\title{
A mixture of mulberry and silk amino acids protected against D-galactosamine induced acute liver damage by attenuating oxidative stress and inflammation in HepG2 cells and rats
}

\author{
SUNMIN PARK, TING ZHANG, XUANGAO WU and JING YI QIU \\ Department of Food and Nutrition, Obesity/Diabetes Center, \\ Hoseo University, Asan, Chungcheong 336-795, Republic of Korea
}

Received January 31, 2019; Accepted October 23, 2019

DOI: $10.3892 /$ etm.2020.8636

\begin{abstract}
The liver is an important organ for the removal of toxins and utilization of nutrients. The present study then investigated whether a mixture of mulberry water extracts and silk amino acids protected against acute liver damage in rats induced by intraperitoneal injection of D-galactosamine and the action mechanism. D-galactosamine injection is widely used to develop experimental animal models of acute hepatic disease. In the present study, male Sprague-Dawley rats received intraperitoneal injection of D-galactosamine followed by 200 and $600 \mathrm{mg} / \mathrm{kg}$ body weight (BW) of mulberry extracts and silk amino acids (1:3, w/w; MS1:3-L and MS1:3-H), the same amounts of MS with different ratios (1:5, w/w; MS1:5-L and MS1:5-H), and $600 \mathrm{mg} / \mathrm{kg}$ bw cellulose (control) for 1 week. The normal-control group received an injection of saline instead of D-galactosamine with the same diet as the control group. D-galactosamine injection (control rats) increased serum ALT, AST and $\gamma$-GPT levels, indicating the induction of acute liver damage. The control rats also exhibited reduced glycogen depositions, which contributed to increasing fat synthesis from glucose and elevated serum triglyceride levels. Oxidative stress and inflammation in the liver of the control increased in response to the decreasing antioxidant activity and mRNA expression and increasing TNF- $\alpha$ expression, respectively. Both MS1:3 and MS1:5 reduced serum ALT, AST and $\gamma$-GPT levels to ameliorate liver damage. MS1:3 reduced oxidative stress by increasing the activity and expression of antioxidant enzymes, whereas MS1:5 decreased the expression TNF- $\alpha$ in the liver. MS1:3 and MS1:5 improved the necrosis of hepatocytes in H\&E staining, which was associated with increased glycogen deposition in PAS staining. MS1:5 had better effects on glycogen accumulation. In
\end{abstract}

Correspondence to: Dr Sunmin Park, Department of Food and Nutrition, Obesity/Diabetes Center, Hoseo University, 165 Sechul-Ri, Asan, Chungcheong 336-795, Republic of Korea E-mail: smpark@hoseo.edu

Key words: D-galactosamine, liver damage, glycogen deposition, necrosis, inflammation, oxidative stress conclusion, MS1:3 and MS1:5 can be used as therapeutic agents for acute liver damage.

\section{Introduction}

The liver, which is the first organ to deliver nutrients and toxins from the intestines through the portal vein after consumption, is responsible for various biological process including metabolizing nutrients and toxic compounds such as alcohol and drugs (1). Since the liver plays a crucial role in eliminating toxic compounds, it is susceptible to damage by toxins such as alcohol, bacteria, and viruses (1). The insults from the toxins can induce acute liver damage via increased oxidative stress and inflammation, and this can then progress to hepatitis, hepatic cancer, and liver cirrhosis (2). Accordingly, acute liver damage needs to be treated at an early stage and it is important to explore effective therapeutic strategies to recover from or protect against acute liver damage.

Hepatic damage is induced by various chemicals including alcohol, bacteria, and viruses. Several chemicals including carbon tetrachloride $\left(\mathrm{CCl}_{4}\right)$, D-galactose, and lipopolysaccharide have been used to induce acute hepatic damage in experimental animal models $(1,3,4)$. Among these chemicals, D-galactosamine derived from D-galactose works as a hepatotoxic compound to induce hepatocy te necrosis and inflammation. D-galactosamine decreases the cellular uridine-5'-triphosphate (UTP) concentration and reduces the synthesis of hepatocyte RNA to block transcription in the liver (5). D-galactosamine injection results in a condition that resembles human viral hepatitis. D-galactosamine is generally accepted for production of animal models for acute liver damage because of its good reproducibility and easy dosage control (3).

Mulberry (Morus alba L.) fruits contain anthocyanins, which have anti-oxidant and anti-inflammation properties. Mulberry fruit extracts have been reported to protect against liver damage caused by tetrachloride, lipopolysaccharide, and oxidative stress $(2,6,7)$. In addition, mulberry extracts improve alcohol-induced steatosis by alleviating gut microbiota (4). The amelioration of liver damage is mainly associated with reduction of oxidative stress and inflammation via changes in the gut microbiota $(4,6)$. Silk amino acids have been shown to have anti-inflammatory activities and to accelerate the 
removal of toxins such as alcohol and acetaldehyde (8). Thus, both mulberry extracts and silk amino acids protect against acute liver damage caused by toxins and they may alleviate acute liver damage additively or synergistically. In the present study, we hypothesized that a mixture of mulberry water extracts and silk amino acids may protect against acute liver damage in rats. This hypothesis was tested in rats treated with intraperitoneal injection of D-galactosamine and the action mechanism was explored. We found that D-galactosamine acutely induced liver injury with decreased glycogen deposition and increased triglyceride accumulation in the liver. The mixture of mulberry and silk amino acids ameliorated liver damage by potentiating anti-oxidant enzymes and reducing proinflammatory cytokines.

\section{Materials and methods}

Effects of HepG2 cell line on cell damage. Human hepatocellular carcinoma HepG2 cells were acquired from American Type Culture Collection (HB-8065) were grown and maintained with high glucose Dulbecco's modified Eagle's medium (DMEM) containing 10\% fetal bovine serum (FBS) (9). Cells were then transferred into 96 -well plates at $4 \times 10^{3}$ cells/well in high-glucose DMEM containing $0.3 \%$ bovine serum albumin (BSA) and allowed to grow to $70 \%$ confluence. The cells were then treated with vehicle, 10 or $50 \mu \mathrm{g} / \mathrm{ml}$ mulberry extracts (MB), silk amino acids (SA) or their mixtures (MS1:2, MS1:3, MS1:4, and MS1:5). After $1 \mathrm{~h}$ of treatment, $50 \mathrm{mM}$ D-galactosamine was added to the cells and they were incubated for another $8 \mathrm{~h}$. The cell viability was then measured in HepG2 cells by 3-(4,5-dimethylthiazol-2-yl)-2,5-diphenyl tetrazolium bromide (MTT) assay using an Aureon plate reader (Aureon Biosystems).

HepG2 cells were grown in 24-well plates at $6 \times 10^{4}$ cells/well until the cells were $70 \%$ confluent, then treated with vehicle, 10 or $50 \mu \mathrm{g} \mathrm{ml}^{-1}$ mulberry extracts (MB), silk amino acids (SA) or their mixtures (MS1:2, MS1:3, MS1:4, and MS1:5) for $1 \mathrm{~h}$. The cells were subsequently treated with $50 \mathrm{mmol} / 1$ D-galactosamine and incubated for $8 \mathrm{~h}$. Next, cells were harvested with lysis buffer at $4^{\circ} \mathrm{C}$ and centrifuged at $10,000 \mathrm{xg}$ for $20 \mathrm{~min}$ at $4^{\circ} \mathrm{C}$, which was followed by collection of the supernatants. Lipid peroxide levels as malondialdehyde (MDA) contents in the liver were then measured using a thiobarbituric acid reactive substance (TBARS) assay kit (Cayman Chemical). Relative mRNA levels of tumor necrosis factor- $\alpha$ $(\mathrm{TNF}-\alpha)$ were measured by real-time polymerase chain reaction (PCR) in samples treated with vehicle, $50 \mu \mathrm{g} \mathrm{ml}^{-1}$ mulberry extracts (MB), silk amino acids (SA) or their mixtures (MS1:2, MS1:3, MS1:4, and MS1:5).

Animal care and experimental design. Male Sprague Dawley rats that weighed an average of $235 \pm 12 \mathrm{~g}$ were purchased from Daehan Bio, Inc. and acclimated in the animal facility. All rats were raised in individual stainless steel cages in a controlled environment $\left(23^{\circ} \mathrm{C}, 12\right.$-h light/dark cycle) and had ad libitum access to food and water. All study procedures were adopted based on the Guide for the Care and Use of Laboratory Animals of the National Institutes of Health (NIH) and were approved by the Institutional Animal Care and Use Committee of Hoseo University (HSIACUC-17-068).
After 1-week of acclimation in the animal facility, 50 male rats received a single intraperitoneal injection of D-galactosamine saline solution $(800 \mathrm{mg} / \mathrm{kg}$ bw) to induce acute liver damage. The rats were then randomly divided into five groups: 1) low dosage ( $200 \mathrm{mg} / \mathrm{kg}$ body weight) of mulberry extracts and silk amino acid (1:3, MS1:3-L, or $1: 5$, MS1:5-L), 2) high dosage (600 mg/kg body weight) of mulberry extracts and silk amino acid $(1: 3, \mathrm{MS} 1: 3-\mathrm{H}$, or 1:5, MS1:5-H) and 3) cellulose (control). Ten rats received a single intraperitoneal injection of saline solution. Animals were given free access to water and 40 energy percent diet containing mulberry extracts and silk amino acid during the 1-week experimental period.

At the end of the experiment, epididymal and retroperitoneal fat mass and uteruses were removed and weighed after rats were sacrificed after anesthesia by subcutaneous injection of a mixture of ketamine and xylazine (100 and $10 \mathrm{mg} / \mathrm{kg}$ body weight, respectively). After blood collection, human insulin ( $5 \mathrm{U} / \mathrm{kg}$ body weight) was injected through the inferior vena cava to determine insulin signaling in the liver. Serum samples were then stored at $-70^{\circ} \mathrm{C}$ for biochemical analysis. Serum was separated after centrifugation of the blood and the serum levels of alanine aminotransferase (ALT), aspartate aminotransferase (AST) and $\gamma$-glutamyl transpeptidase $(\gamma$-GPT), which are markers for liver damage, were measured by colorimetric methods using kits obtained from Asan Pharmaceutical company (Seoul, Korea). Livers were collected and stored at $-70^{\circ} \mathrm{C}$ for further study.

The livers were homogenized with $1.5 \mathrm{~N}$ perchloric acid and the lysates were treated with $\alpha$-amyloglucosidase to hydrolyze glycogen. The hydrolysate was then neutralized with $\mathrm{NaOH}$ to $\mathrm{pH} 7.4$ and centrifuged at $3000 \mathrm{rpm}$ for $10 \mathrm{~min}$, after which the glucose concentration was measured using a glucose oxidase kit (Young Dong Pharm.) and liver glycogen was calculated from the glucose concentrations. Triacylglycerol was extracted from the livers and brains with chloroform-methanol (2:1, $\mathrm{vol} / \mathrm{vol}$ ) and resuspended in pure chloroform. After evaporating chloroform, the residues were suspended with PBS containing $0.1 \%$ triton X-100 and then sonicated and boiled for $5 \mathrm{~min}$. Finally, the triacylglycerol contents of the suspensions were assayed using a Trinder kit (Young Dong Pharm.) (10).

Oxidative stress status. Lipid peroxide levels as MDA in the liver were measured using a TBARS assay kit (Cayman Chemical). Triglyceride (TG) contents were also measured in the liver and brain using a TG kit (Asan Pharmaceutical). TNF- $\alpha$ levels in the liver lysate were measured using ELISA kits (R \& D Systems, Minneapolis, MN and Amersham Biosciences, Piscataway, NJ, USA). The activities of antioxidant enzymes such as $\mathrm{Cu} / \mathrm{Zn}$ superoxide dismutase (SOD1) and glutathione (GSH)-peroxidase were determined from the lysates of liver tissues using colorimetry kits (Cayman Chemical and Biovision), respectively (11). One unit of each enzyme activity was defined as 50\% inhibition of each enzyme reaction and the enzyme activity was normalized by $\mathrm{mg}$ protein in the lysate. Additionally, GSH levels in the liver were determined using a GSH assay kit (Sigma-Aldrich; Merck KGaA).

Isolation of liver total RNA and real-time PCR. Livers were powdered with a cold steel mortar and pestle, then mixed with 
a monophasic solution of phenol and guanidine isothiocyanate (TRIzol reagent; Gibco; Thermo Fisher Scientific, Inc.) for total RNA extraction, which was conducted according to the manufacturer's instructions. cDNA was synthesized from equal amounts of total RNA using superscript III reverse transcriptase, and polymerase chain reaction (PCR) was performed with high-fidelity Taq DNA polymerase. Equal amounts of cDNA were added to SYBR Green mix (BioRad Laboratories, Inc.) and amplified using a real-time PCR instrument (Bio-Rad Laboratories, Inc.). The expression levels of the genes of interest were normalized to those of the housekeeping gene $\beta$-actin. To assess changes in the expression of genes related to fatty acid synthesis and oxidation in the liver, the following primers were used: tumor necrosis factor (TNF)- $\alpha$ (forward 5'-CCCTGGTACTAACTCCCA GAAA-3', reverse 5'-TGTATGAGAGGGACGGAACC-3'), interleukin (IL)-1 $\beta$ (forward 5'-CACCTCTCAAGCAGAGCA CAG-3', reverse 5'-GGGTTCCATGGTGAAGTCAAC-3'), $\mathrm{Cu} / \mathrm{Zn}$ superoxide dismutase (SOD; forward 5'-CACTCT AAGAAACATGGCG-3', reverse 5'-CTGAGAGTGAGA TCACACG-3'), GSH-Px (forward 5'-GCGGGCCCTGGC ATTG-3', reverse 5'-GGACCAGCGCCCATCTG-3') and $\beta$-actin (forward 5'-AGCGTGGCTACAGCTTCACC-3', reverse 5'-AAGTCTAGGGCAACATAGCACAGC-3'). The relative gene expression was quantified using the comparative cycle of threshold (CT) method (2- ${ }^{-\Delta \Delta \mathrm{Cq}}$ method) as previously described by Livak and Schmittgen (12).

Histological analysis. At the end of the experimental period, the liver samples were fixed in $10 \%$ buffered neutral formaldehyde and embedded in paraffin wax. Histological sections that were $6 \mu \mathrm{m}$ thick were stained with hematoxylin and eosin (H\&E) and used to score the liver damage. The liver damage and glycogen contents were determined in two sections with a random selection from six consecutive sections at $\times 200$ magnification. The liver damage was scored by summing each item such as the nucleus size and shape, cell size and arrangement and the number of macrophages in the histological scoring system. Each item was scored as 0 (no change), 1 (mid), 2 (moderate) and 3 (severe) (4). Higher scores indicated more hepatic cell damage. In addition, glycogen contents were determined by the red color intensity in periodic acid-Schiff (PAS) staining of the stomach tissues. Lower scores indicated higher glycogen contents (4).

Statistical analysis. Statistical analysis was performed with SAS software version 7 (SAS Institute, Inc.) and the results are expressed as the means \pm standard deviation. The variables with results from different time points were analyzed with one-way ANOVA to assess the metabolic effects of the mixture of mulberry extracts and silk amino acids in male rats fed a high-fat diet. Multiple comparisons between groups were identified by Tukey's test at $\mathrm{P}<0.05$.

\section{Results}

Cell viability of HepG2 cell line damaged by $D$-galactosamine. As shown in Fig. 1A, D-galactosamine reduced cell viability in $\mathrm{HepG} 2$ cells and high dosage pretreatment of SA and MB protected against the cell

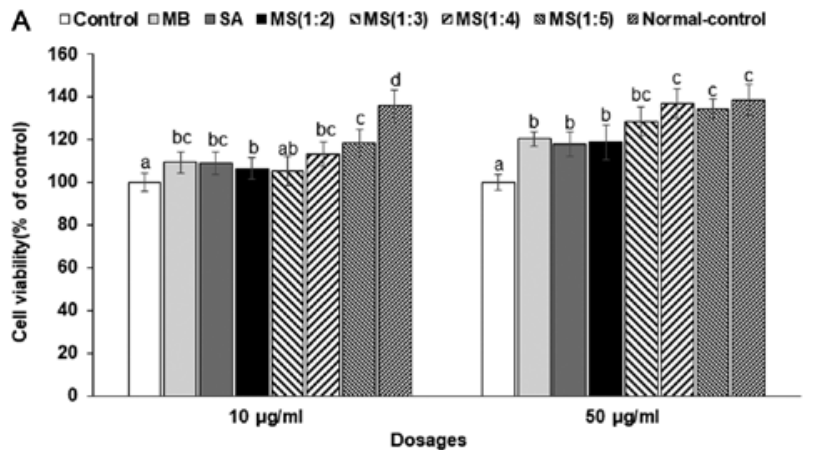

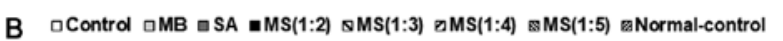

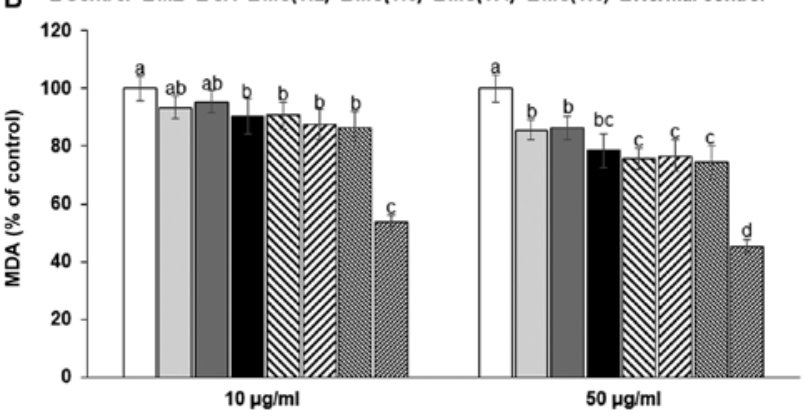

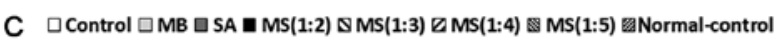

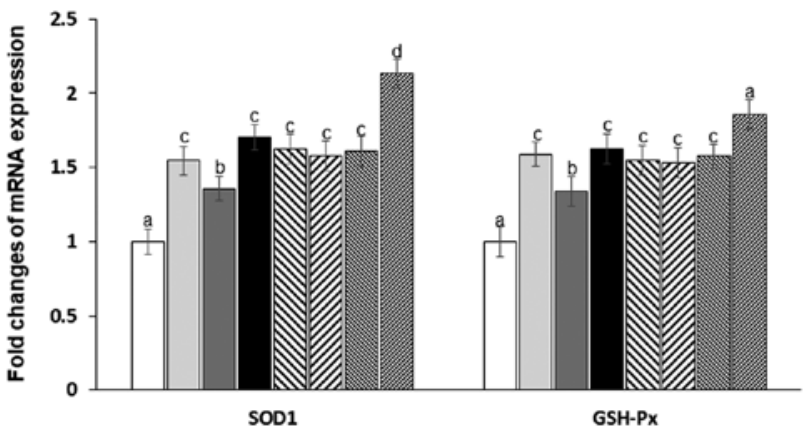

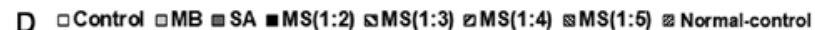

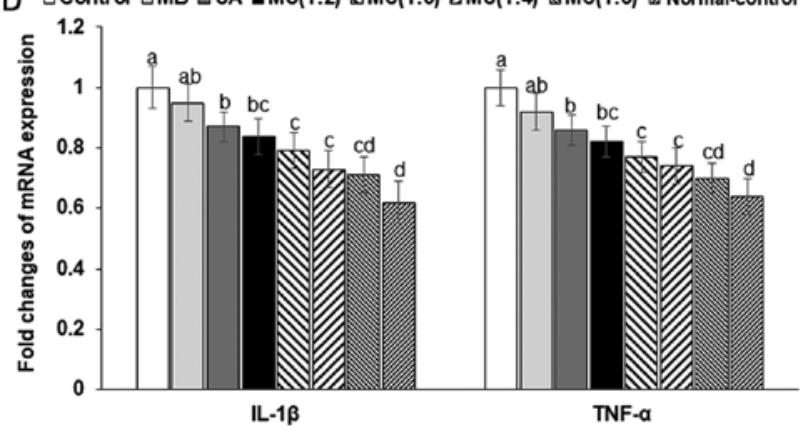

Figure 1. The effects of mulberry water extracts and silk amino acids on HepG2 cells damaged by D-galactosamine. HepG2 cells were treated with 0 (vehicle), 10 or $50 \mu \mathrm{g} / \mathrm{ml} \mathrm{MB}, \mathrm{SA}$ or their mixtures (MS1:2, MS1:3, MS1:4, and MS1:5). After $1 \mathrm{~h}$ of treatment, $50 \mathrm{mM} \mathrm{D}$-galactosamine was added to the cells and they were incubated for an additional $24 \mathrm{~h}$. (A) Cell viability measured by MTT assays. (B) Lipid peroxide levels measured as MDA. (C) Fold changes of SOD1 and GSH-Px mRNA expression. (D) Fold changes of IL-1 $\beta$ and TNF- $\alpha$ mRNA expression. ${ }^{*} \mathrm{P}<0.05,{ }^{* *} \mathrm{P}<0.01$ and ${ }^{* * *} \mathrm{P}<0.001$ vs. Control. MB, mulberry extracts; SA, silk amino acids; MDA, malondialdehyde; SOD1, superoxide dismutase 1; GSH-Px, glutathione peroxidase; IL-1 $\beta$, interleukin-1 $\beta$; TNF- $\alpha$, tumor necrosis factor- $\alpha$. $\mathrm{b} \mathrm{P}<0.05, \mathrm{c} \mathrm{P}<0.01$ and $\mathrm{dP}<0.001$ vs. Control.

damage caused by D-galactosamine. The mixture of MS1:4 and MS1:5 improved the cell viability in D-galactosamine intoxicated HepG2 cells. 
Table I. Energy metabolism and mulberry and silk amino acid intake.

\begin{tabular}{lccccccc}
\hline Variables & $\begin{array}{c}\text { Control } \\
(\mathrm{n}=10)\end{array}$ & $\begin{array}{c}\text { MS1:3-L } \\
(\mathrm{n}=10)\end{array}$ & $\begin{array}{c}\text { MS1:3-H } \\
(\mathrm{n}=10)\end{array}$ & $\begin{array}{c}\text { MS1:5-L } \\
(\mathrm{n}=10)\end{array}$ & $\begin{array}{c}\text { MS1:5-H } \\
(\mathrm{n}=10)\end{array}$ & $\begin{array}{c}\text { Normal-control } \\
(\mathrm{n}=10)\end{array}$ & P-value $^{\mathrm{a}}$ \\
\hline Body weight at 1 week (g) & $262 \pm 18$ & $256 \pm 17$ & $257 \pm 18$ & $248 \pm 20$ & $250 \pm 19$ & $260 \pm 1$ & 0.28 \\
Caloric intake (kcal/day) & $70.9 \pm 7.4$ & $67.1 \pm 6.8$ & $68.7 \pm 6.2$ & $65.7 \pm 6.6$ & $68.4 \pm 7.1$ & $68.1 \pm 4.8$ & 0.17 \\
MB intake (mg/day) & $0 \pm 0$ & $16.5 \pm 1.7^{\mathrm{c}}$ & $50.9 \pm 5.1^{\mathrm{d}}$ & $9.5 \pm 1.1^{\mathrm{b}}$ & $30.7 \pm 2.5^{\mathrm{d}}$ & $0 \pm 0$ & $<0.001$ \\
SA intake (mg/day) & $0 \pm 0$ & $33.0 \pm 3.5^{\mathrm{d}}$ & $102 \pm 10^{\mathrm{d}}$ & $40.3 \pm 4.2^{\mathrm{d}}$ & $122 \pm 13^{\mathrm{d}}$ & $0 \pm 0$ & $<0.001$ \\
Liver weight (weight \%) & $4.3 \pm 0.1$ & $4.1 \pm 0.2$ & $3.8 \pm 0.2^{\mathrm{b}}$ & $4.2 \pm 0.2$ & $4.1 \pm 0.2$ & $3.7 \pm 0.1^{\mathrm{b}}$ & 0.03 \\
\hline
\end{tabular}

${ }^{\text {aP }}$-value of each parameter by one-way ANOVA among the groups. ${ }^{\mathrm{b}} \mathrm{P}<0.05,{ }^{\mathrm{c}} \mathrm{P}<0.01$ and ${ }^{\mathrm{d}} \mathrm{P}<0.001$ vs. Control. Values are means \pm standard deviation. Rats had an intraperitoneal injection of D-galactosamine and they were provided 200 and $600 \mathrm{mg} / \mathrm{kg}$ body weight of mulberry extracts and silk amino acid (1:3, w/w; MS1:3-L and MS1:3-H), the same amounts of MS with different ratio (1:5, w/w; MS1:5-L and MS1:5-H), and $300 \mathrm{mg} / \mathrm{kg}$ bw cellulose (control) for 1 week. The normal-control rats were injected with saline instead of D-galactosamine and they had the same diet with the control group.

Oxidative stress and inflammation in HepG2 cell line. The cell damage was associated with increased oxidative stress. Specifically, the MDA contents in HepG2 cells were 2-fold higher in the HepG2 cells damaged with D-galactosamine (control) than in the normal-controls (Fig. 1B). However, SA and MB reduced the contents of MDA and lipid peroxide index induced by D-galactosamine, with the high dosage mixture of SA and MB leading to greater decreases than either compound alone.

Decreased MDA was related to increased anti-oxidant enzyme expression. The SOD1 and GSH-Px expression were 2- and 1.7-fold lower, respectively, in the control group than the normal-control group (Fig. 1C). However, MB and SA $(50 \mu \mathrm{g} / \mathrm{ml})$ increased SOD1 and GSH-Px expression, with MB leading to greater expression of both enzymes than SA. The mixture of MS1:2, MS1:3, MS1:4 and MS1:5 elevated their expression to levels similar to MB.

However, the expression of both proinflammatory cytokines (IL-1 $\beta$ and TNF- $\alpha$ ) was 1.7-fold higher in the control than the normal-control. SA protected against the increase of IL- $1 \beta$ and TNF- $\alpha$ expression better than MB and the mixture of $\mathrm{MB}$ and SA reduced their expression (Fig. 1D). MS1:5 decreased the expression of IL- $1 \beta$ and TNF- $\alpha$ as much as the normal-control.

Energy metabolism in rats. Treatment with D-galactosamine for one week did not change body weight. In addition, although MS treatments tended to lower body weight, no significant differences were observed among groups (Table I). Additionally, there were no significant differences in energy intake between control and normal-control groups and MS treatment did not alter energy intake, indicating that D-galactosamine and MS treatments did not alter food intake (Table I). MB and SA intake were dependent on the food intake and the ratio and dosage of $\mathrm{MB}$ and SA in the foods. Although body weight was not significantly different between control and normal-control groups, liver weight based on the body weight was higher in the control than the normal-control group (Table I). MS1:3-H suppressed the increase of liver weight in rats injected with D-galactosamine.

Liver damage in rats. Serum AST and ALT levels, which are indexes of liver damage, were higher in the control group than

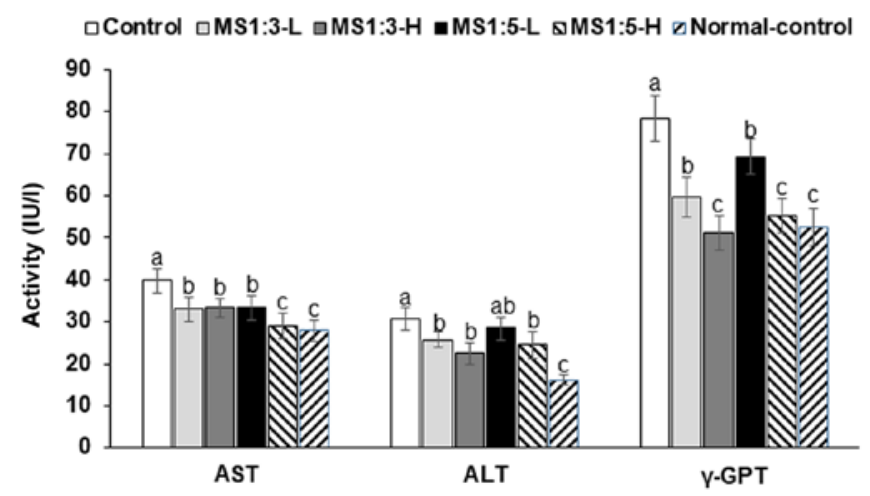

Figure 2. Serum ALT, AST and $\gamma$-GPT levels in rats. Values are the means \pm standard deviation. Rats received intraperitoneal injection of D-galactosamine and were provided with 200 and $600 \mathrm{mg} / \mathrm{kg}$ body weight of mulberry extracts and silk amino acid (1:3, w/w; MS1:3-L and MS1:3-H), the same amounts of MS with silk amino acid at different ratios $(1: 5, \mathrm{w} / \mathrm{w}$; MS1:5-L and MS1:5-H), or $600 \mathrm{mg} / \mathrm{kg}$ bw cellulose (control) for 1 week. Normal-control rats were injected with saline instead of D-galactosamine and had the same diet as the control group. ${ }^{*} \mathrm{P}<0.05$ and ${ }^{* *} \mathrm{P}<0.01$ vs. Control. ALT, alanine aminotransferase; AST, aspartate aminotransferase; $\gamma$-GPT, $\gamma$-glutamyl transferase. $\mathrm{b} \mathrm{P}<0.05, \mathrm{c} \mathrm{P}<0.01$ and $\mathrm{dP}<0.001$ vs. Control.

the normal-control group (Fig. 2). However, MS1:3 and MS1:5 suppressed these increases in a dose-dependent fashion. MS1:3 suppressed the decrease in serum ALT and AST levels more than MS1:5. In addition, serum $\gamma$-GPT levels showed the same patterns as serum AST and ALT levels. Additionally, serum TNF- $\alpha$ levels were about 2-fold higher in the control than the normal-control group (Table II), but MS1:3 and MS1:5 inhibited this increase. Taken together, these findings indicate MS1:3 and MS1:5 reduced the liver damage induced by D-galactosamine dose-dependently.

The liver plays a crucial role in lipid metabolism and liver damage alters the metabolism. Serum total, high-density lipoprotein (HDL) and low-density lipoprotein (LDL) cholesterol levels were not significantly different following treatment with D-galactosamine and MS (Table II). However, serum triglyceride levels were elevated by D-galactosamine injection and reduced by MS treatment. Treatment with MS1:5 decreased serum triglyceride levels compared to MS1:3 (Table II). In 
Table II. Lipid profiles and glucose metabolism in the circulation.

\begin{tabular}{lccccccc}
\hline Variables & $\begin{array}{c}\text { Control } \\
(\mathrm{n}=10)\end{array}$ & $\begin{array}{c}\text { MS1:3-L } \\
(\mathrm{n}=10)\end{array}$ & $\begin{array}{c}\text { MS1:3-H } \\
(\mathrm{n}=10)\end{array}$ & $\begin{array}{c}\text { MS1:5-L } \\
(\mathrm{n}=10)\end{array}$ & $\begin{array}{c}\text { MS1:5-H } \\
(\mathrm{n}=10)\end{array}$ & $\begin{array}{c}\text { Normal-control } \\
(\mathrm{n}=10)\end{array}$ & P-value $^{\mathrm{a}}$ \\
\hline Serum TNF- $\alpha(\mathrm{pg} / \mathrm{ml})$ & $57.6 \pm 4.7$ & $44.6 \pm 4.9^{\mathrm{b}}$ & $38.2 \pm 4.1^{\mathrm{c}}$ & $36.2 \pm 4.3^{\mathrm{c}}$ & $31.2 \pm 3.4^{\mathrm{d}}$ & $23.1 \pm 2.9^{\mathrm{d}}$ & 0.004 \\
Serum total cholesterol $(\mathrm{mg} / \mathrm{dl})$ & $108 \pm 9.8$ & $99.7 \pm 8.2$ & $97.3 \pm 6.8$ & $99.3 \pm 9.7$ & $101 \pm 8.6$ & $96.9 \pm 7.3$ & 0.07 \\
Serum HDL-C (mg/dl) & $18.7 \pm 1.7$ & $19.7 \pm 1.8$ & $19.0 \pm 1.5$ & $18.5 \pm 1.6$ & $19.7 \pm 1.8$ & $18.5 \pm 1.5$ & 0.09 \\
Serum LDL-C (mg/dl) & $63.8 \pm 5.9$ & $58.0 \pm 5.5$ & $58.7 \pm 5.6$ & $64.9 \pm 6.7$ & $65.2 \pm 6.2$ & $61.2 \pm 5.8$ & 0.08 \\
Serum triglyceride (mg/dl) & $127 \pm 11.4$ & $110 \pm 10.7^{\mathrm{b}}$ & $97.8 \pm 9.4^{\mathrm{b}}$ & $79.1 \pm 8.4^{\mathrm{c}}$ & $80.7 \pm 7.6^{\mathrm{c}}$ & $83.2 \pm 7.1^{\mathrm{c}}$ & 0.01 \\
Serum glucose (mg/dl) & $139.5 \pm 8.3$ & $127.6 \pm 8.1^{\mathrm{b}}$ & $126.9 \pm 7.6^{\mathrm{b}}$ & $115.7 \pm 7.2^{\mathrm{b}}$ & $118.9 \pm 6.8^{\mathrm{b}}$ & $130.7 \pm 7.9^{\mathrm{b}}$ & 0.02 \\
Serum insulin $(\mathrm{pg} / \mathrm{ml})$ & $1.97 \pm 0.16$ & $1.69 \pm 0.17^{\mathrm{b}}$ & $1.74 \pm 0.15^{\mathrm{b}}$ & $1.61 \pm 0.14^{\mathrm{b}}$ & $1.56 \pm 0.17^{\mathrm{b}}$ & $1.83 \pm 0.15^{\mathrm{b}}$ & 0.03 \\
HOMA-IR & $11.0 \pm 1.0$ & $8.6 \pm 0.8^{\mathrm{b}}$ & $8.8 \pm 0.7^{\mathrm{b}}$ & $7.5 \pm 0.6^{\mathrm{c}}$ & $7.4 \pm 0.6^{\mathrm{c}}$ & $9.6 \pm 0.9^{\mathrm{b}}$ & 0.01 \\
\hline
\end{tabular}

${ }^{\text {aP }}$ value of each parameter by one-way ANOVA among the groups. ${ }^{\mathrm{b}} \mathrm{P}<0.05,{ }^{\mathrm{c}} \mathrm{P}<0.01$ and ${ }^{\mathrm{d}} \mathrm{P}<0.001$ vs. Control. Values are means \pm standard deviation. Rats had an intraperitoneal injection of D-galactosamine and they were provided 200 and $600 \mathrm{mg} / \mathrm{kg}$ body weight of mulberry extracts and silk amino acid (1:3, w/w; MS1:3-L and MS1:3-H), the same amounts of MS with different ratio (1:5, w/w; MS1:5-L and MS1:5-H), and $600 \mathrm{mg} / \mathrm{kg}$ bw cellulose (control) for 1 week. The normal-control rats were injected with saline instead of D-galactosamine and they had the same diet with the control group. TNF- $\alpha$, tumor necrosis factor- $\alpha$; HDL-C, high density lipoprotein cholesterol; LDL-C, low density lipoprotein; HOMA-IR, homeostatic model assessment for insulin resistance.

addition, triglyceride deposition in the liver was elevated in the control group compared to the normal-control group and MS1:3 and MS1:5 inhibited this increase (Table III). In contrast, glycogen deposition in the liver was lower in the control than the normal-control and MS1:3 and MS1:5 prevented the decrease induced by D-galactosamine injection (Table III). Thus, D-galactosamine injection damaged the liver tissues, which resulted in disturbance of triglyceride metabolism but not cholesterol metabolism.

Liver histology in rats. H\&E staining showed that D-galactosamine disrupted the hepatocytes (Fig. 3A). The nuclei of the hepatocytes were enlarged, the cellular arrangement was disrupted and the macrophage infiltration was elevated compared to the control (Fig. 3A and B). The results indicated that D-galactose induced hepatocyte damage. MS1:3 and MS1:5 protected against hepatocyte damage (Fig. 3B). Macrophage infiltration was also reduced by MS1:3 and MS1:5 (Fig. 3B).

D-galactosamine depleted glycogen deposition in the control group in comparison to the normal-control (Fig. 3B, C). MS1:3 and MS1:5 increased the glycogen deposition and MS1:5-H increased glycogen deposition more than the normal-control (Fig. 3B and C).

Oxidative stress in the liver of the rats. The amount of MDA, an index of lipid peroxide, was higher in the control group than the normal-control group (Table III). The activity of SOD, which is an enzyme that removes superoxide, was lower in the control group than the normal-control group. MS1:3 dose-dependently increased the SOD activity as did MS1:5. In parallel with SOD, GSH-Px activity was also lower in the control than the normal-control and MA1:5 suppressed this decrease (Table III). Evaluation of the hepatic level of GSH revealed that the substrate of GSH-Px was lower in the control than the normal-control, indicating that lower levels of reducing agents increased lipid peroxides in the control than the normal-control (Table III). MS1:3 better inhibited the decrease of hepatic GSH activities than MS1:5. Hepatic levels of TNF- $\alpha$ were more than 2-fold higher in the control than the normal-control and they decreased more in response to MS1:5 than MS1:3 (Table III).

In addition to the activity and contents of anti-oxidant enzymes and proinflammatory cytokines, their mRNA expression was influenced by D-galactosamine and MS mixtures modified the mRNA expression (Fig. 4). The SOD1 and GSH-Px mRNA expression were 1.5-fold lower in the control than the normal-control, while MS1:3 increased their expression (Fig. 4). The mRNA expression of IL-1 $\beta$ and TNF- $\alpha$, proinflammatory cytokines was higher in the control than the normal-control, while MS1:3 and MS1:5 lowered their expression (Fig. 4). MS1:5-H decreased their mRNA expression similar to the normal-control.

\section{Discussion}

The liver plays a crucial role in removal of toxins and utilization of nutrients to regulate glucose and lipid metabolism. Toxins such as $\mathrm{CCL}_{4}$ and D-galactosamine induce acute liver damage and disturb lipid and glucose metabolism $(2,5,13)$. D-galactosamine is known to induce a condition similar to human viral hepatitis and is therefore widely used to generate experimental animal models of the acute hepatic disease. The etiology of D-galactosamine to induce acute liver damage is associated with depleting UTP levels in the liver (5), stimulating proinflammatory cytokines including IL-1 $\beta$ and TNF- $\alpha$ (14) and elevating oxidative stress by reducing antioxidants (15). Depletion of UTP levels may be involved in glycogen synthesis in the liver; thus, like other toxins such as alcohol, D-galactosamine induces acute hepatocyte necrosis toxicity and changes the glucose and lipid metabolism. We investigated whether mixtures of mulberry water extracts and silk amino acids could protect against acute liver damage in rats induced by intraperitoneal injection of D-galactosamine and the mechanism of the identified protective action. The 

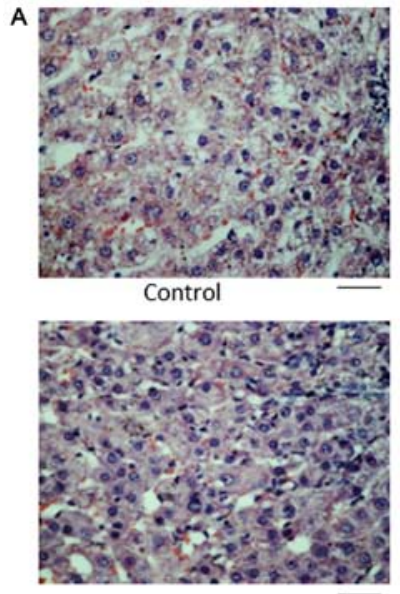

MS1:5-L

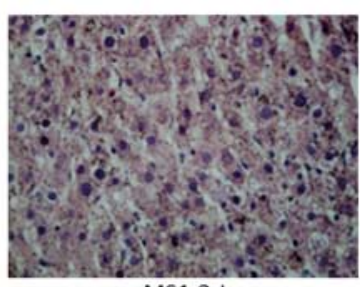

MS1:3-L

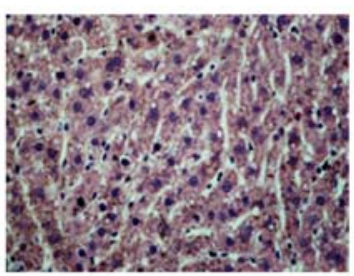

MS1:5-H

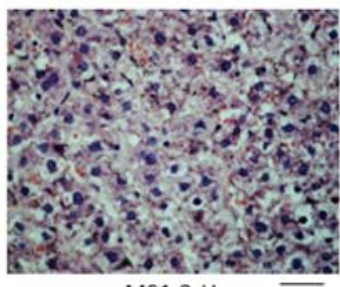

MS1:3-H

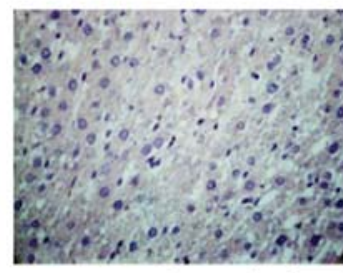

Normal-control

B 口Control $\square M S 1: 3-L \quad \square M S 1: 3-H \quad \square M S 1: 5-L \quad \triangle M S 1: 5-H \quad \nabla N o r m a l-c o n t r o l$

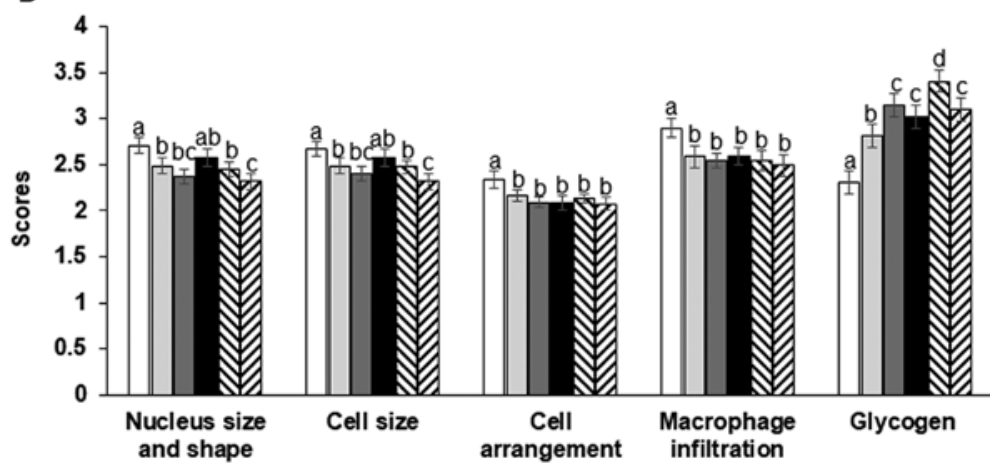

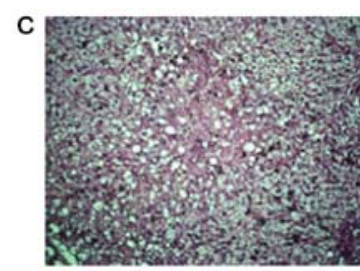

Control

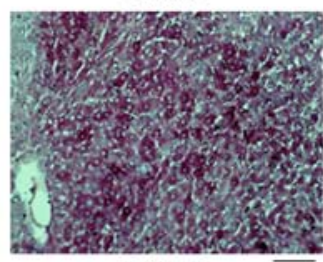

MS1:5-L

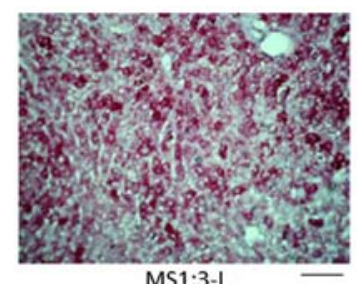

MS1:3-L

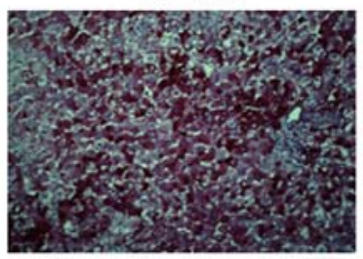

MS1:5-H

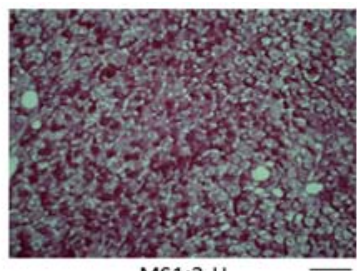

MS1:3-H

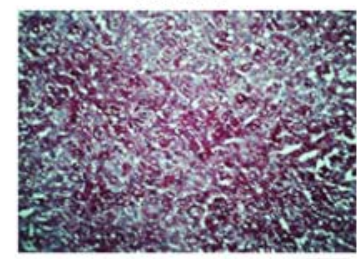

Normal-control

Figure 3. Liver histology with H\&E and PAS staining. (A) Liver section stained with H\&E. (B) Liver section stained with PAS. (C) Scores of cell disruption. Values are the means \pm standard deviation. Rats received an intraperitoneal injection of D-galactosamine and were provided with $200 \mathrm{and} 600 \mathrm{mg} / \mathrm{kg}$ body weight of mulberry extracts and silk amino acid (1:3, w/w; MS1:3-L and MS1:3-H), the same amounts of MS with silk amino acid at different ratios (1:5, w/w; MS1:5-L and MS1:5-H), or $600 \mathrm{mg} / \mathrm{kg}$ bw cellulose (control) for 1 week. The normal-control rats were injected with saline instead of D-galactosamine and had the same diet as the control group. 200x original magnification. Scale bar, $50 \mu \mathrm{m}$. " $\mathrm{P}<0.05$ and ${ }^{* *} \mathrm{P}<0.01$ vs. Control. H\&E, hematoxylin and eosin; PAS, periodic acid-schiff. b $\mathrm{P}<0.05, \mathrm{c} \mathrm{P}<0.01$ and $\mathrm{dP}<0.001$ vs. Control.

results showed that D-galactosamine induced acute liver damage was attenuated by a mixture of silk amino acid and mulberry water extracts by reducing oxidative stress and inflammation.

Silk amino acids are derived from the hydrolysis of cocoons from the silkworm Bombyx mori. Silk amino acids contain $75 \%$ fibroin and $25 \%$ sericin, and intake of silk amino acids, including sericin hydrolysate, has been shown to exert protection against alcohol-induced liver damage by accelerating alcohol elimination through urine directly, which enhanced the oxidation rate in the liver $(8,16)$. Thus, silk amino acid intake reduces ethanol concentration in the liver and in circulation and may therefore reduce liver damage; however, it may not ameliorate liver damage induced by other toxins besides ethanol. In the present study, mulberry water extracts were combined with silk amino acid to protect against D-galactosamine induced liver damage in rats. The various ratios of mulberry water extracts and silk amino acids were then examined to improve cell survival in HepG2 cells treated with D-galactosamine. 
Table III. Hepatic lipid peroxides, antioxidant enzyme activities, and triglyceride and glycogen deposition.

\begin{tabular}{|c|c|c|c|c|c|c|c|}
\hline Variables & $\begin{array}{l}\text { Control } \\
(n=10)\end{array}$ & $\begin{array}{l}\text { MS1:3-L } \\
(\mathrm{n}=10)\end{array}$ & $\begin{array}{l}\text { MS1:3-H } \\
\quad(n=10)\end{array}$ & $\begin{array}{l}\text { MS1:5-L } \\
(\mathrm{n}=10)\end{array}$ & $\begin{array}{l}\text { MS1:5-H } \\
(\mathrm{n}=10)\end{array}$ & $\begin{array}{l}\text { Normal-control } \\
\qquad(\mathrm{n}=10)\end{array}$ & P-value \\
\hline Liver TG (mg/g tissue) & $88.6 \pm 5.9$ & $82.8 \pm 5.3^{b}$ & $81.2 \pm 4.6^{\mathrm{b}}$ & $86.2 \pm 4.6$ & $82.3 \pm 6.2^{b}$ & $79.3 \pm 5.8^{b}$ & 0.04 \\
\hline Liver glycogen (mg/g tissue) & $22.2 \pm 3.6$ & $33.7 \pm 5.1^{\mathrm{c}}$ & $31.1 \pm 4.8^{c}$ & $34.5 \pm 4.5^{\mathrm{c}}$ & $32.1 \pm 5.1^{\mathrm{c}}$ & $38.1 \pm 4.0^{\mathrm{d}}$ & 0.001 \\
\hline Hepatic MDA (nmol/mg protein) & $0.96 \pm 0.09$ & $0.75 \pm 0.08^{\mathrm{b}}$ & $0.68 \pm 0.07^{\mathrm{c}}$ & $0.85 \pm 0.08^{\mathrm{b}}$ & $0.74 \pm 0.08^{\mathrm{b}}$ & $0.61 \pm 0.08^{c}$ & 0.01 \\
\hline Hepatic SOD (U/mg protein) & $25.6 \pm 3.2$ & $31.9 \pm 3.9^{b}$ & $39.3 \pm 4.1^{\mathrm{c}}$ & $27.8 \pm 3.4$ & $31.4 \pm 3.5^{\mathrm{b}}$ & $36.6 \pm 3.8^{b}$ & 0.01 \\
\hline Hepatic GSH peroxide (U/mg protein) & $66.8 \pm 6.5$ & $72.6 \pm 7.1$ & $79.6 \pm 8.3^{\mathrm{b}}$ & $68.9 \pm 7.1$ & $75.1 \pm 6.7^{\mathrm{b}}$ & $84.5 \pm 8.8^{b}$ & 0.02 \\
\hline Hepatic GSH (umol/g protein) & $21.1 \pm 2.3$ & $26.4 \pm 2.4^{\mathrm{b}}$ & $29.3 \pm 2.5^{\mathrm{b}}$ & $24.1 \pm 2.7$ & $26.2 \pm 2.3^{\mathrm{b}}$ & $29.4 \pm 2.5^{\mathrm{b}}$ & 0.02 \\
\hline Hepatic TNF- $\alpha$ (pg/g tissue) & $9.5 \pm 0.9$ & $8.7 \pm 0.9$ & $7.6 \pm 0.7^{\mathrm{b}}$ & $8.0 \pm 0.9^{b}$ & $6.8 \pm 0.7^{b}$ & $4.1 \pm 0.5^{\mathrm{c}}$ & 0.004 \\
\hline
\end{tabular}

${ }^{\text {aP }}$-value of each parameter by one-way ANOVA among the groups. ${ }^{\mathrm{b}} \mathrm{P}<0.05,{ }^{\mathrm{c}} \mathrm{P}<0.01$ and ${ }^{\mathrm{d}} \mathrm{P}<0.001$ vs. Control. Values are means \pm standard deviation. Rats had an intraperitoneal injection of D-galactosamine and they were provided 200 and $600 \mathrm{mg} / \mathrm{kg}$ body weight of mulberry extracts and silk amino acid (1:3, w/w; MS1:3-L and MS1:3-H), the same amounts of MS with different ratio (1:5, w/w; MS1:5-L and MS1:5-H), and $600 \mathrm{mg} / \mathrm{kg}$ bw cellulose (control) for 1 week. The normal-control rats were injected with saline instead of D-galactosamine and they had the same diet with the control group. TG, triglyceride; MDA, malondialdehyde; SOD, superoxide dismutase; GSH, glutathione; TNF- $\alpha$, tumor necrosis factor- $\alpha$.

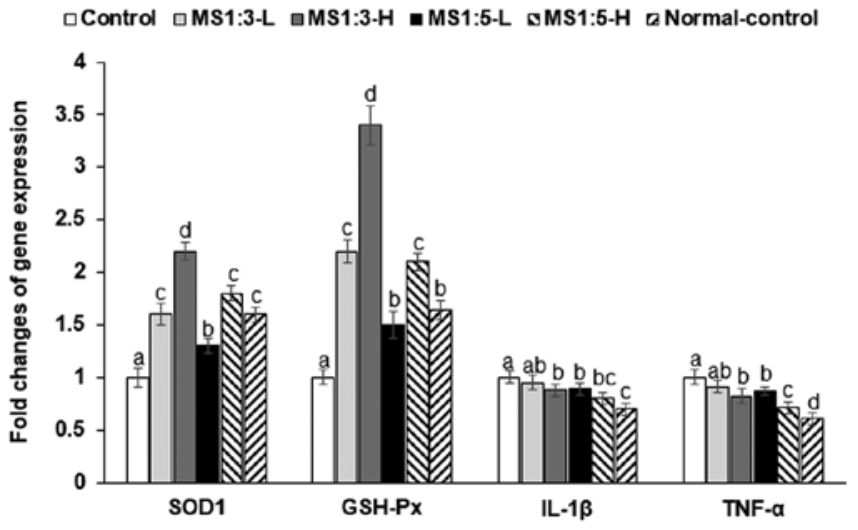

Figure 4. mRNA expression of SOD1, GSH-Px, and TNF- $\alpha$. Values are the means \pm standard deviation. Rats received an intraperitoneal injection of D-galactosamine and were provided with 200 and $600 \mathrm{mg} / \mathrm{kg}$ body weight of mulberry extracts and silk amino acid (1:3, w/w; MS1:3-L and MS1:3-H), the same amounts of MS with silk amino acid at different ratios (1:5, w/w; MS1:5-L and MS1:5-H), or $600 \mathrm{mg} / \mathrm{kg}$ bw cellulose (control) for 1 week. The normal-control rats were injected with saline instead of D-galactosamine and they had the same diet as the control group. ${ }^{*} \mathrm{P}<0.05,{ }^{* * *} \mathrm{P}<0.01$ and ${ }^{* * *} \mathrm{P}<0.001$ vs. Control. SOD1, superoxide dismutase-1; GSH-Px, glutathione peroxidase; TNF- $\alpha$, tumor-necrosis factor- $\alpha$. $\mathrm{b} \mathrm{P}<0.05$, c $\mathrm{P}<0.01$ and $\mathrm{dP}<0.001$ vs. Control.

Mulberry water extracts have been reported to contain anthocyanins such as cyanidin-3-glucoside as well as flavonoids such as rutin, quercetin, and luteolin (8); therefore, they can act as antioxidants. Mulberry water extracts have been demonstrated to protect against ethanol-induced liver disease by reducing inflammation and inhibiting lipogenesis in the liver $(8,17)$. In addition, mulberry water extracts have been shown to ameliorate obesity or $\mathrm{CCL}_{4}$-induced liver damage by decreasing lipid peroxidation and inhibiting proinflammatory gene expression $(2,13)$. The results of the present study showed that high dosages of MA and SA themselves increased cell survival when liver damage was induced by D-galactosamine. However, the mixture of MA and SA elevated cell survival more than either extract alone. The mixture of MS reduced the MDA contents with increased SOD1 and GSH-Px expression in the HepG2 cells treated with D-galactosamine in HepG2 cells. Additionally, the levels of TNF- $\alpha$, which indicate inflammation, were lower samples treated with high doses of MA and SA; however, the mixture of MS1:5 led to greater decreases than either individual extract. Thus, the MS mixture, especially MS1:5, may ameliorate acute liver damage better than individual $\mathrm{MB}$ and SA.

In the present study, rats fed MS1:3 and MS 1:5 protected against acute liver injury by D-galactosamine. As shown in other studies, D-galactosamine injection induced liver damage by increasing oxidative stress and decreasing the activity of antioxidants including SOD1 and GSH-Px and their mRNA expression and inflammation by increasing proinflammatory cytokines such as TNF- $\alpha$. The MS1:3 and MS1:5 decreased oxidative stress and inflammation. MS1:3 decreased lipid peroxide levels in the liver by elevating the activities and mRNA expression of antioxidant enzymes including SOD1 and GSH-Px more than MS1:5. However, MS1:5 reduced proinflammatory cytokines such as TNF- $\alpha$ better than MS1:3. As a result, both MS1:3 and MS1:5 improved the hepatic damage based on histology and reduced liver damage indexes such as serum AST and ALT. Serum AST and ALT activities were much lower in the present study, which measured them 7 days after D-galactosamine injection as opposed to $18-24 \mathrm{~h}$ in the previous studies (18-20). The previous studies have showed substantial variations in their activities (18-20). In the present study, serum AST and ALT activities in the control were much higher than the normal-control and MS1:3 and MS1:5 treatments decreased the activities compared to the control. The decrease in AST and ALT was largely a time dependent improvement in which the D-galactosamine-induced liver damage was naturally alleviated in all groups including the control, the improvement was accelerated by MS1:3 and MS1:5.

Mulberry extract may act as an antioxidant to reduce oxidative stress and improve inflammation as shown in 
other studies (6-8). Silk amino acids have been reported to improve glucose metabolism by increasing insulin secretion and pancreatic $\beta$-cell mass, even in type 1 diabetes $(21,22)$, suggesting that they have the potential to improve the recovery of damaged cells. Thus, silk amino acids may improve the recovery of damaged hepatocytes by D-galactosamine.

No previous studies has reported that glycogen deposition was reduced and TG accumulation was elevated in the livers of the rats with D-galactosamine induced acute liver damage. This was associated with the ability of D-galactosamine to reduce UTP levels in the liver since UTP is associated with the synthesis of RNA in the hepatocytes and glycogen synthesis (5). Cell damage by D-galactosamine may be associated with the inhibition of protein production and glycogen synthesis in the liver. Although glycogen storage disease type 1a caused by the deficiency of glucose- 6 phosphatase is associated with decreasing glycogen utilization to excessively accumulate not only glycogen storage but also triglycerides in the liver, it has progressed to steatohepatitis, cirrhosis and hepatic adenomas and carcinomas (23). However, depletion of glycogen has been reported to be related to hepatocyte damage with insulin resistance by environmental toxins $(24,25)$. Moreover, decreases in glycogen deposition have been shown to contribute to increasing synthesis of fat from glucose and to exacerbate glucose metabolism to increase insulin secretion to normalize serum glucose levels, but not to decrease serum glucose levels and increase insulin resistance (26). The present study showed that rats in the control had increased HOMA-IR and insulin resistance and developed glucose intolerance. In addition, serum triglyceride levels increased in the control group relative to the MS1:3 and MS1:5 treatments, even though the serum total, HDL and LDL cholesterol levels did not differ among groups. D-galactosamine may not influence cholesterol metabolism in the liver and MS1:3 and MS1:5 did not alter the cholesterol metabolism. Glucose intolerance, insulin resistance and serum triglyceride levels induced by D-galactosamine were alleviated by both MS1:3 and MS1:5 and they were improved better in MS1:5 than MS1:3. These results suggested that MS1:5 recovered hepatic function, especially glucose and lipid metabolism, better than MS1:3 although both MS1:3 and MS1:5 led to similar improvements in oxidative stress and inflammation. Therefore, MS1:5 may be used as a therapeutic agent for acute liver damage induced by $\mathrm{D}$-galactosamine.

In conclusion, D-galactosamine injection induced acute hepatic damage by increasing oxidative stress and inflammation as well as the accumulation of decreased glycogen and increased triglyceride levels. However, both MS1:3 and MS1:5 reduced serum ALT, AST and $\gamma$-GPT levels to ameliorate liver damage, with MS1:5 having better effects on glycogen and triglyceride accumulation. Therefore, MS1:5 may be a good therapeutic agent for acute liver damage. Low and high doses of MS1:3 and MS1:5 of 1.5 and $4.5 \mathrm{~g} /$ day, respectively, were applied as human equivalents.

\section{Acknowledgements}

Not applicable.

\section{Funding}

This study was financially supported by the Ministry of Trade, Industry, and Energy (MOTIE), Korea, under the 'Regional Specialized Industry Development Program' (grant no. R0006422) supervised by the Korea Institute for Advancement of Technology (KIAT) and the Traditional Culture Convergence Research Program through the National Research Foundation of Korea (NRF) funded by the Ministry of Science and ICT (grant no. NRF-2016M3C1B5907049).

\section{Availability of data and materials}

The datasets used and/or analyzed during the current study are available from the corresponding author on reasonable request.

\section{Authors' contributions}

SP designed the study and wrote the manuscript. TZ and XW conducted biochemical experiments. TZ, JYQ and XW conducted the animal study and biochemical assays. All authors reviewed the manuscript and approved the submission.

\section{Ethics approval and consent to participate}

The current study was approved by the Institutional Animal Care and Use Committee of Hoseo University (approval no. HSIACUC-17-068).

\section{Patient consent for publication}

Not applicable.

\section{Competing interests}

The authors declare that they have no competing interests.

\section{References}

1. Lim DW, Kim H, Park JY, Kim JE, Moon JY, Park SD and Park WH: Amomum cardamomum L. ethyl acetate fraction protects against carbon tetrachloride-induced liver injury via an antioxidant mechanism in rats. BMC Complement Altern Med 16: 155, 2016.

2. Hsu LS, Ho HH, Lin MC, Chyau CC, Peng JS and Wang CJ: Mulberry water extracts (MWEs) ameliorated carbon tetrachloride-induced liver damages in rat. Food Chem Toxicol 50: 3086-3093, 2012.

3. Radhiga T, Sundaresan A, Viswanathan P and Pugalendi KV: Effect of protocatechuic acid on lipid profile and DNA damage in D-galactosamine-induced hepatotoxic rats. J Basic Clin Physiol Pharmacol 27: 505-514, 2016.

4. Park S, Kim DS, Wu X and Q JY: Mulberry and dandelion water extracts prevent alcohol-induced steatosis with alleviating gut microbiome dysbiosis. Exp Biol Med (Maywood) 243: 882-894, 2018.

5. Decker K and Keppler D: Galactosamine hepatitis: Key role of the nucleotide deficiency period in the pathogenesis of cell injury and cell death. Rev Physiol Biochem Pharmacol: 77-106, 1974.

6. Ou TT, Kuo CY, Chyau CC, Lee HJ, Peng JS and Wang CJ: Improvement of lipopolysaccharide-induced hepatic injuries and inflammation with mulberry extracts. J Sci Food Agric 93: 1880-1886, 2013.

7. Yan F, Chen Y, Azat R and Zheng X: Mulberry anthocyanin extract ameliorates oxidative damage in HepG2 cells and prolongs the lifespan of Caenorhabditis elegans through MAPK and Nrf2 pathways. Oxid Med Cell Longev 2017: $7956158,2017$. 
8. Yang HJ, Kim MJ, Kang ES, Kim DS and Park S: Red mulberry fruit aqueous extract and silk proteins accelerate acute ethanol metabolism and promote the antioxidant enzyme systems in rats. Mol Med Rep 18: 1197-1205, 2018.

9. Lopez-Terrada D, Cheung SW, Finegold MJ and Knowles BB: Hep G2 is a hepatoblastoma-derived cell line. Hum Pathol 40: $1512-1515,2009$.

10. Jeong SY, Kang S, Hua CS, Ting Z and Park S: Synbiotic effects of beta-glucans from cauliflower mushroom and Lactobacillus fermentum on metabolic changes and gut microbiome in estrogen-deficient rats. Genes Nutr 12: 31, 2017.

11. Moon NR, Kang S and Park S: Consumption of ellagic acid and dihydromyricetin synergistically protects against UV-B induced photoaging, possibly by activating both TGF- $\beta 1$ and wnt signaling pathways. J Photochem Photobiol B 178: 92-100, 2018.

12. Livak KJ and Schmittgen TD: Analysis of relative gene expression data using real-time quantitative PCR and the 2(-Delta Delta C(T)) method. Methods 25: 402-408, 2001

13. Lim HH, Lee SO, Kim SY, Yang SJ and Lim Y: Anti-inflammatory and antiobesity effects of mulberry leaf and fruit extract on high fat diet-induced obesity. Exp Biol Med (Maywood) 238: $1160-1169,2013$

14. Ganai AA, Khan AA, Malik ZA and Farooqi H: Genistein modulates the expression of NF-KB and MAPK (P-38 and ERK1/2), thereby attenuating d-Galactosamine induced fulminant hepatic failure in Wistar rats. Toxicol Appl Pharmacol 283: 139-146, 2015.

15. Sakaguchi S and Yokota K: Role of $\mathrm{Ca} 2+$ on endotoxin-sensitivity by galactosamine challenge: Lipid peroxide formation and hepatotoxicity in zymosan-primed mice. Pharmacol Toxicol 77: 81-86, 1995.

16. Li YG, Ji DF, Chen S and Hu GY: Protective effects of sericin protein on alcohol-mediated liver damage in mice. Alcohol Alcohol 43: 246-253, 2008.

17. Tang CC,Huang HP,Lee YJ,Tang YHandWang CJ:Hepatoprotective effect of mulberry water extracts on ethanol-induced liver injury via anti-inflammation and inhibition of lipogenesis in C57BL/6J mice. Food Chem Toxicol 62: 786-796, 2013.
18. Saracyn M, Zdanowski R, Brytan M, Kade G, Nowak Z, Patera J, Dyrla P, Gil J and Wankowicz Z: D-galactosamine intoxication in experimental animals: Is it only an experimental model of acute liver failure? Med Sci Monit 21: 1469-1477, 2015.

19. Ganai AA and Husain M: Genistein attenuates D-GalN induced liver fibrosis/chronic liver damage in rats by blocking the TGF- $\beta /$ Smad signaling pathways. Chem Biol Interact 261: 80-85, 2017.

20. Banu S, Bhaskar B and Balasekar P: Hepatoprotective and antioxidant activity of Leucas aspera against D-galactosamine induced liver damage in rats. Pharm Biol 50: 1592-1595, 2012.

21. Do SG, Park JH, Nam H, Kim JB, Lee JY, Oh YS and Suh JG: Silk fibroin hydrolysate exerts an anti-diabetic effect by increasing pancreatic $\beta$ cell mass in $\mathrm{C} 57 \mathrm{BL} / \mathrm{KsJ}-\mathrm{db} / \mathrm{db}$ mice. J Vet Sci 13 : 339-344, 2012.

22. Song Z, Zhang M, Xue R, Cao G and Gong C: Reducing blood glucose levels in TIDM mice with an orally administered extract of sericin from hIGF-I-transgenic silkworm cocoons. Food Chem Toxicol 67: 249-254, 2014.

23. Farah BL, Sinha RA, Wu Y, Singh BK, Lim A, Hirayama M, Landau DJ, Bay BH, Koeberl DD and Yen PM: Hepatic mitochondrial dysfunction is a feature of Glycogen Storage Disease Type Ia (GSDIa). Sci Rep 7: 44408, 2017.

24. Henrique da Silva G, Barros PP, Silva Goncalves GM and Landi MA: Hepatoprotective effect of Lycopodium clavatum $30 \mathrm{CH}$ on experimental model of paracetamol-induced liver damage in rats. Homeopathy 104: 29-35, 2015.

25. Irimia JM, Meyer CM, Segvich DM, Surendran S, DePaoli-Roach AA, Morral N and Roach PJ: Lack of liver glycogen causes hepatic insulin resistance and steatosis in mice. J Biol Chem 292: 10455-10464, 2017.

26. Crescenzo R, Bianco F, Mazzoli A, Giacco A, Liverini G and Iossa S: A possible link between hepatic mitochondrial dysfunction and diet-induced insulin resistance. Eur J Nutr 55: 1-6, 2016

This work is licensed under a Creative Commons Attribution-NonCommercial-NoDerivatives 4.0 International (CC BY-NC-ND 4.0) License. 